\title{
SOFTWARE FOR ADAPTING DSPZ RECEIVERS TO THE URAN INTERFEROMETER NETWORK
}

\author{
E.A.Isaeva ${ }^{1}$, O.A.Lytvynenko ${ }^{1}$, V.A.Shepelev ${ }^{2}$ \\ ${ }^{1}$ URAN-4 Observatory of IRA NASU, Odessa, Ukraine, uran4@te.net.ua \\ ${ }^{2}$ Institute of Radio Astronomy, NASU, Kharkov, Ukraine, shep@rian.kharkov.ua
}

ABSTRACT. More than 10 years ago, URAN interferometer network (Megn A.V.,1997; Konovalenko A.A., 2014) had been equipped with newly designed receivers with a pass band extended up to $250 \mathrm{kHz}$ and software rejection of interferences (Rashkovskii, 2012). The broadening of bandwidth of received signal increase the sensitivity of the receivers significantly and let us to investigate the angular structure about one hundred radio sources. A software package had been developed that allows: preparing a program of observations, carrying out observations automatically, making data cross-correlation, calculating visibility functions for all pairs of antennae, and fitting models of an angular structure of the sources. Data storage formats were elaborated for each stage of recording or processing.

At present, new digital radio astronomy receiver DSPZ have been developed by IRA NASU (Zakharenko, 2016). The receiver allows recording an entire bandwidth of signals of a decameter range from 8 to $32 \mathrm{MHz}$. It is used at UTR-2 and URAN radio telescopes operated in a single dish mode. Application of the receivers for interferometer observation with the URAN network provides additional advantages in accuracy and sensitivity of studies. In this report we consider the data formats and synchronization methods used in URAN equipment and DSPZ receivers, and discuss algorithms of their transformation. Newly elaborated software is described, that allows selecting a set of frequency bands of signals recorded with DSPZ and converting them to the form used by the URAN software. This approach allows us to carry out the interferometer observations in an the extended frequency range provided by DSPZ and to use as much as possible the software package developed for the URAN network for data reduction.

Keywords: URAN network, interferometer observation, DSPZ receiver, frequency bands, time marker

\section{Introduction}

The UTR-2 radio telescope is currently equipped with digital DSPZ receivers with a wide dynamic range determined by 16-bit ADCs. They allow to record all frequency range of signals received by the radio telescope 8 to 30 $\mathrm{MHz}$. These receivers are very versatile and can be used in various observational programs, including interferometric studies.
URAN interferometers used set of receivers with narrower pass band which operate simultaneously at two selected frequencies of the range.

Extending of the pass band and / or increasing the number of operating frequencies using DSPZ would be very useful for improvement of the quality of the interferometer studies of radio sources structure. A software package for data correlation and reduction has been worked out for the URAN earlier and using of these developments will accelerate application of the DSPZ receivers to interferometer studies.

The purpose of this work is to create software for conversion of data collected by DSPZ broadband receivers to the format used in the URAN. It makes possible to carry out the data reduction using the software elaborated for these interferometers.

\section{Date formats and conversion data files}

The data recorded by DSPZ receiver are stored in files of $*$.jds format. The format of data recorded by URAN receiver is $*$.itm. The difference of signals recorded by the receivers is presented in the Table 1 . Therefore a structure of data stored, service information, and synchronization methods used in these formats are quite different.

Table 1: data parameters of two formats

\begin{tabular}{|l|c|c|}
\hline \multicolumn{1}{|c|}{ Parameters } & Format *.jds & Format *.itm \\
\hline Signal bandwidth, $\mathrm{MHz}$ & 16,33 & 0,25 \\
Sampling frequency, $\mathrm{MHz}$ & 33,66 & 1 \\
ADC resolution, bits & 16 & 8 \\
Output channels & 2 & 4 \\
\hline
\end{tabular}

Let's consider a structure of a computer program developed to transform of the data stored in *.jds data files recorded by DSPZ to format of *.itm data files used by the URAN receiver. The program consists of two independent modules: dsp_transform.exe and dsp_fft.sav. The main module dsp_transform.exe is written in Delphi while IDL is used for called program module dsp_fft.sav. The program module dsp_transform.exe contains a user interface to manage the data conversion program and visualize process of conversion. 


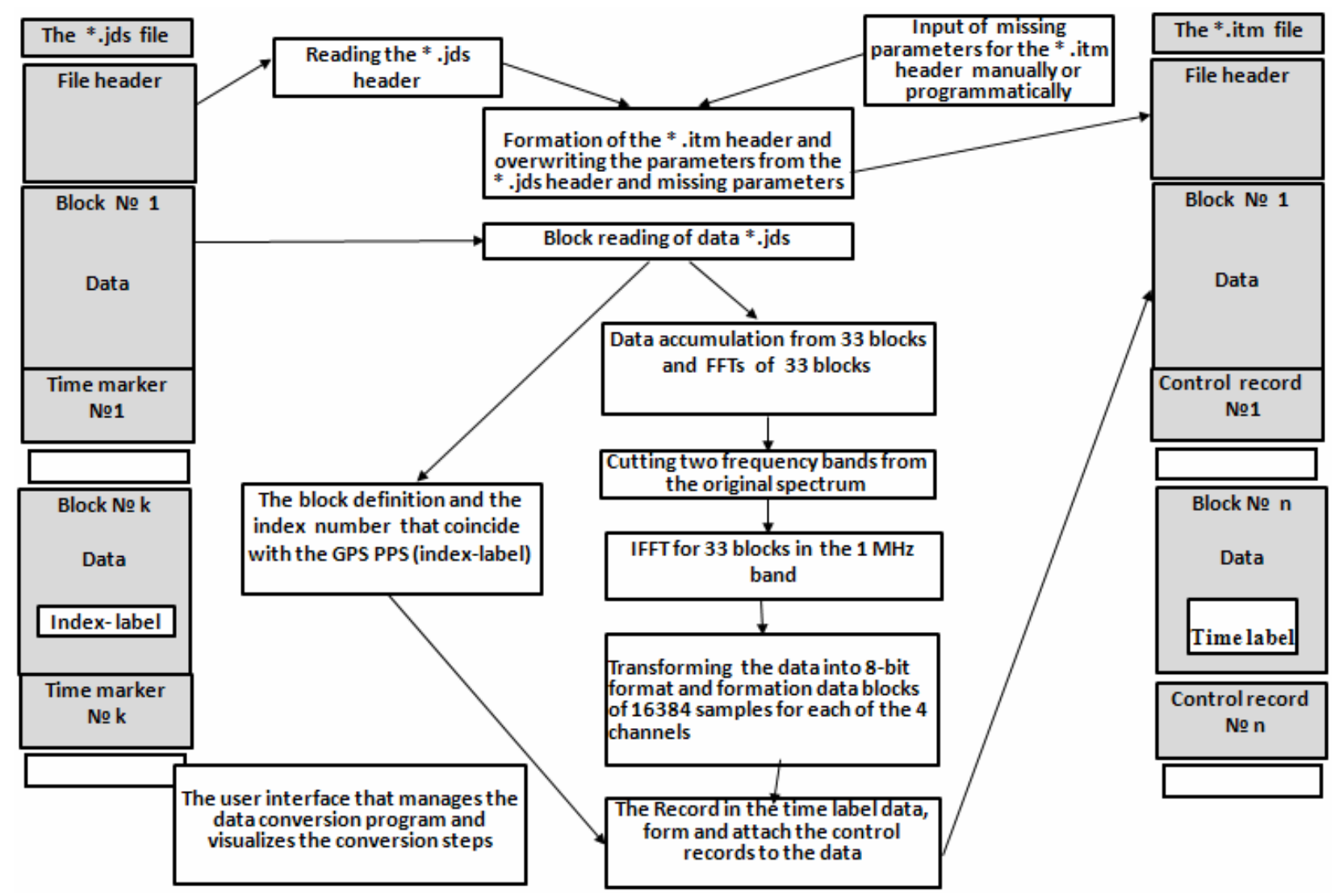

Figure 1: Diagram of the data conversion by the program dsp_transform.exe

The program module dsp_fft.sav allows to transform data recorded in $*$.jds format to $*$.itm format.

In general, conversion of data in $*$.jds format to $*$.itm format can be represented as the following scheme (see Figure 1).

\section{Spectral transformations}

For radio interferometer observations with DSPZ receivers, the frequency band of the input signal can be limited to range from $16,5 \mathrm{MHz}$ to $30 \mathrm{MHz}$. In this case, the Nyquist condition will be satisfied if the sampling frequency of DSPZ is $33 \mathrm{MHz}$. That range contains operating frequencys of URAN receivers (usually 20 and $25 \mathrm{MHz}$ ).

After performing of the Fast Fourier Transform (FFT) of the DSPZ receiver output signal, an inverted spectrum in the range $0-16.5 \mathrm{MHz}$ will be obtained. In this case it is necessary to consider that the current frequency $\mathrm{f}$ in the spectrum of the original signal corresponds to the current frequency $\mathrm{f}_{\mathrm{FFT}}=33 \mathrm{MHz}-\mathrm{f}$ in the inverted spectrum (see Figure $2 \mathrm{a}$ ). Thus, in order to obtain the original spectrum, it is necessary to invert the indexes of counts in the obtained inverted spectrum. Figure 2 shows the results of signal transform of the DSPZ receiver test record (from *.jds format to *.itm format).

The next step of format conversion is extracting of narrow bands from the original spectrum and reducing sampling frequency. These narrow bands should correspond to the band of recorded signals in *.itm files. The bandwidth of the URAN receiver recorded signal is $250 \mathrm{kHz}$, and the sampling frequency of a signal is $1 \mathrm{MHz}$.

The second procedure after FFT is cutting out two band of $1 \mathrm{MHz}$ width from the inverted spectrum of the original DSPZ signal.

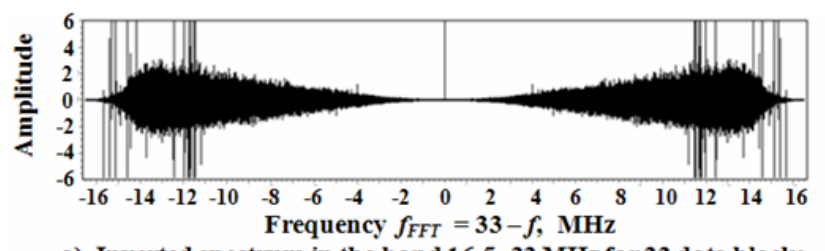

a). Inverted spectrum in the band $16.5-33 \mathrm{MHz}$ for 33 data blocks

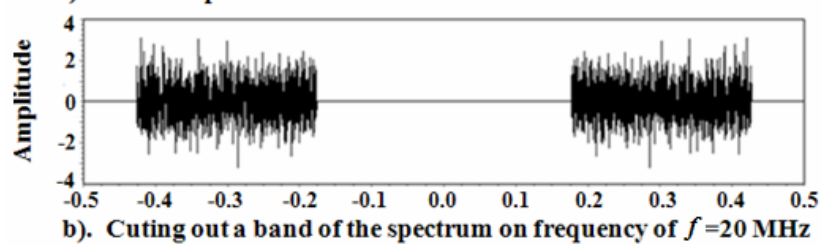

b). Cuting out a band of the spectrum on frequency of $f=20 \mathrm{MHz}$ a width of $\Delta f=250 \mathrm{kHz}$

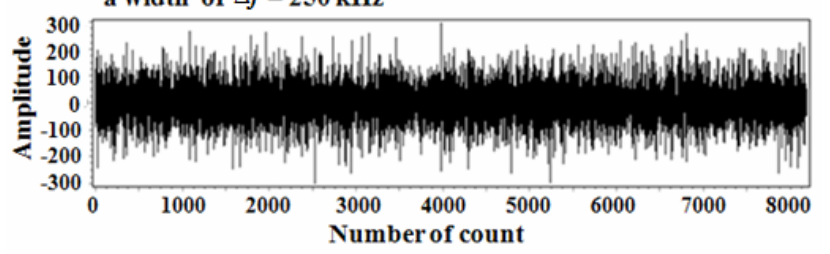

c). The inverse Fourier transform, $f=20 \mathrm{MHz}, \Delta f=250 \mathrm{kHz}$ The data is given to a sampling frequency of $1 \mathrm{MHz}$

Figure 2: Cutting the frequency band and converting the data to the new sampling frequency. 
Each of bands must include one of the operating frequencies of the URAN receivers. In extracted band, all spectral components must be zeroed apart from those corresponding to the recorded signal of the URAN receiver.

This procedure of cutting out the band of the spectrum from the data in the *.jds format automatically reduces the sampling rate by 33 times, which corresponds to a sampling frequency in the *.itm format $(1 \mathrm{MHz})$. Figure $2 \mathrm{~b}$ shows an example of cutting out and shifting a $250 \mathrm{kHz}$ band with a center frequency $\mathrm{f}=20 \mathrm{MHz}$, which corresponds to $\mathrm{f}_{\mathrm{FFT}}=13 \mathrm{MHz}$ in inverted spectrum.

Then, using the Inverse Fourier Transform (IFFT) of the extracted frequency band, we obtain a signal in the *.itm format. Figure $3 \mathrm{c}$ shows a signal in the *.itm format which was obtained from the DSPZ receiver test record.

\section{Formation of data blocks and their synchronization}

The data in the formats $*$.jds and *.itm differ in the size of the data blocks, the number of channels, and the service information recorded at the end of each block, and also the ADC resolution.

The data block in *.itm format has the dimension equal to array $[i=0 . .16383, j=0 . .3]$, and in the format $*$.jds ar$\operatorname{ray}[i=0 . .8189, j=0 . .1]$, where $i$ is the number of count, and $j$ is the channel number. Thus, the next step of the data conversion is the formation of data blocks of a correct size, which corresponds to *.itm format. Then a control record must be generated for each block and inserted at the end of the block. The control record contains the parameters which are necessary for the data synchronization. These parameters are calculated using an information from time markers of *.jds blocks.

The DSPZ receivers use 16-bit ADCs while the URAN data are stored in 8-bit. For the purpose of transformation, the standard procedure in IDL BYTSCL is used, which allows scaling the signal at specified boundary values. Three options for converting data to an 8-bit format for different situations and tasks are provided.

The first option uses an absolute scaling of the signal over the maximum and minimum amplitude values for the entire record. This option cannot work properly when spikes occur with amplitude which exceeds several times the maximum operating range of a useful signal. In this case the useful signal will be significantly suppressed. The second option is the most universal. It uses a specified boundary values of BYTSCL procedure associated with the roof-mean-square deviation of the signal that was calculated for the entire record. The third option is direct clipping of higher bits of a binary signal. This option can be used when the signal recording mode of DSPZ receivers is limited to 8 bits.

Data in the formats *.jds and *.itm differ in the way of synchronization. In the *.jds format, a time marker is recorded at the end of each data block, containing seconds of the day and a phase of the seconds equal to the number of samples passed since the beginning of each second. Unlike the *.jds format, in *.itm format, the time label is embedded directly into the data block instead of some sample. Thus, knowing the phase of a second in the *.jds format, one can calculate the index of sample in the data stream in the *.itm format, taking into account the reduction in the sampling rate by 33 times. Now you can insert time labels into the generated $*$.itm file.

\section{Conclusion}

The software for the conversion of DSPZ data to the format used in the VLBI URAN has been developed. This software was tested in the long-baseline interferometer observations with the URAN-2 interferometer (Shepelev V.A., 2017). The standard URAN interferometers equipment and DSPZ receivers were used simultaneously in this experiment. Obtained results demonstrate the effectiveness of the application of the programs developed.

\section{References}

Konovalenko A.A., Kalinichenko N.N., Lytvynenko O.A. et al.: 2014, VarSITI_Newsletter, 2, 1.

Megn A.V., Braude S.Y̌a., Rashkovskii S.L. et al.: 1997, Radiophys. and Radioastron., 2, 385 (in Russian).

Rashkovskii S.L., Belov A.S., Ivanov A.S. et al.: 2012, Radiophys. and Radioastron., 17, 201 (in Russian).

Zakharenko V., Konovalenko A., Zarka P. et al.: 2016, J. Astron. Instrum., 5, no. 4. id1641010.

Shepelev V.A., Konovalenko A.A., Lytvynenko O.A. et al.: 2017, in 17-th Odessa International Astronomical Gamow Conference-School Abstracts 2017, Odessa, Ukraine, p. 30. 